\title{
Next generation DNA sequencing technology delivers valuable genetic markers for the genomic orphan legume species, Bituminaria bituminosa
}

María Pazos-Navarro ${ }^{1}$, Mercedes Dabauza', Enrique Correal ${ }^{2}$, Kelly Hanson ${ }^{3}$, Natasha Teakle ${ }^{4,5}$, Daniel Real ${ }^{5,6,7}$ and Matthew N Nelson ${ }^{5^{*}}$

\begin{abstract}
Background: Bituminaria bituminosa is a perennial legume species from the Canary Islands and Mediterranean region that has potential as a drought-tolerant pasture species and as a source of pharmaceutical compounds. Three botanical varieties have previously been identified in this species: albomarginata, bituminosa and crassiuscula. B. bituminosa can be considered a genomic 'orphan' species with very few genomic resources available. New DNA sequencing technologies provide an opportunity to develop high quality molecular markers for such orphan species.
\end{abstract}

Results: 432,306 mRNA molecules were sampled from a leaf transcriptome of a single B. bituminosa plant using Roche 454 pyrosequencing, resulting in an average read length of $345 \mathrm{bp}$ (149.1 Mbp in total). Sequences were assembled into 3,838 isotigs/contigs representing putatively unique gene transcripts. Gene ontology descriptors were identified for 3,419 sequences. Raw sequence reads containing simple sequence repeat (SSR) motifs were identified, and 240 primer pairs flanking these motifs were designed. Of 87 primer pairs developed this way, 75 (86.2\%) successfully amplified primarily single fragments by PCR. Fragment analysis using 20 primer pairs in 79 accessions of B. bituminosa detected 130 alleles at 21 SSR loci. Genetic diversity analyses confirmed that variation at these SSR loci accurately reflected known taxonomic relationships in original collections of B. bituminosa and provided additional evidence that a division of the botanical variety bituminosa into two according to geographical origin (Mediterranean region and Canary Islands) may be appropriate. Evidence of cross-pollination was also found between botanical varieties within a B. bituminosa breeding programme.

Conclusions: B. bituminosa can no longer be considered a genomic orphan species, having now a large (albeit incomplete) repertoire of expressed gene sequences that can serve as a resource for future genetic studies. This experimental approach was effective in developing codominant and polymorphic SSR markers for application in diverse genetic studies. These markers have already given new insight into genetic variation in B. bituminosa, providing evidence that a division of the botanical variety bituminosa may be appropriate. This approach is commended to those seeking to develop useful markers for genomic orphan species.

\footnotetext{
* Correspondence: matthew.nelson@uwa.edu.au

${ }^{5}$ School of Plant Biology, Faculty of Natural and Agricultural Sciences, The University of Western Australia, 35 Stirling Highway, Crawley, WA 6009,

Australia

Full list of author information is available at the end of the article
}

\section{Biomed Central}

(c) 2011 Pazos-Navarro et al; licensee BioMed Central Ltd. This is an Open Access article distributed under the terms of the Creative Commons Attribution License (http://creativecommons.org/licenses/by/2.0), which permits unrestricted use, distribution, and reproduction in any medium, provided the original work is properly cited. 


\section{Background}

Bituminaria bituminosa (L.) C.H. Stirt., commonly known as Tedera in the Canary Islands, is a perennial legume species widely distributed in the Mediterranean Basin and Macaronesia. It is a self-pollinated diploid species $(2 \mathrm{n}=20)$ with DNA content estimated to be between 0.998 and 1.094 pg DNA per diploid nucleus [1-3]. B. bituminosa shows particularly high diversity in the Canary Islands, with three recognised botanical varieties: (i) var. albomarginata: native to semi-arid habitats in coastal areas of Lanzarote island and a few other niches in Fuerteventura, Tenerife and Gran Canaria, with an annual rainfall of $150 \mathrm{~mm}$ to $300 \mathrm{~mm}$; (ii) var. crassiuscula: native to high altitude sub-humid areas in Tenerife island with up to $500 \mathrm{~mm}$ rainfall per year; and (iii) var. bituminosa widely distributed in all islands across varying altitudes and rainfall levels [4,5]. In the Mediterranean basin, only var. bituminosa is found [6]. These botanical varieties were identified using morphological characteristics and were largely supported by preliminary molecular analyses using arbitrary DNA markers [7,8]. However, Juan et al. [7] found that accessions of var. bituminosa from the Mediterranean region formed a cluster distinct from a Canary Islands cluster that contained all three botanic varieties. Therefore, there is some ambiguity in botanical variety definitions that warrants further investigation.

In recent years, there has been growing international interest in B. bituminosa as a potential source of pharmaceutical compounds and also as a drought tolerant pasture species. The plant contains secondary compounds such as pterocarpans with antitumor activity against leukaemia and colon cancer [9-11], antioxidants [12] and furanocoumarins such as psoralen and angelicin, which are used in the treatment of skin diseases (psoriasis, vitiligo, melanoma) [13-15]. As a forage crop it is well adapted to high temperature and low rainfall. An important attribute of this species is that, unlike lucerne (Medicago sativa L.), it has a high retention of leaves when moisture stressed, therefore providing valuable feed over summer $[16,17]$. Traditionally, the profitability and sustainability of livestock industries in southern Australia and in other regions with Mediterranean-like climates is severely constrained by the quantity and quality of forage available over summer and autumn. Therefore, there is strong demand for breeding drought-tolerant and productive forage legumes as well as improved understanding of the genetic basis of key agronomic traits.

Molecular markers contribute valuable support to breeding programmes [18]. Markers provide the means to characterise genetic diversity within breeding programmes and help identify new genetic diversity in the wild or in germplasm collections. Markers are valuable in determining or confirming pedigrees and for markerassisted selection of traits that are difficult and/or expensive to measure. However, B. bituminosa can be considered a true 'genomic orphan' [19] in that there are almost no genomic resources or high-quality codominant markers available for genetic analysis. The few genomic resources readily available for molecular marker development for B. bituminosa consist largely of chloroplast gene sequences developed for phylogenetic studies within the tribe Psoraleeae and more broadly among phaseoloid legumes [20,21]. However, B. bituminosa has a rich cousin in the genomic resources sense: soybean (Glycine max (L.) Merr.) that belongs to the neighbouring subtribe Glycininae [21]. The complete genome sequence of soybean was recently determined [22], which could act as a useful reference genome for B. bituminosa. However, the taxonomic divide between these species is sufficiently wide to make marker transfer between soybean and B. bituminosa rather inefficient. Fortunately, the advent of new high-throughput genome sequencing technologies provides a relatively low cost opportunity for rapid development of locusspecific markers for a species like $B$. bituminosa that has little available genomic resources.

This study reports the generation of a cDNA library developed from leaf mRNA from a single $B$. bituminosa plant and sampling of the leaf transcriptome using 454 GS-FLX pyrosequencing technology. Simple sequence repeat (SSR) motifs were identified, primers designed and a subset of these markers were used to characterise a broad set of B. bituminosa accessions to assist in the correct choice of parents in breeding programmes, and which could be used to provide guidance in managing and conserving germplasm collections. These SSR markers along with the first catalogue of expressed genes provide valuable resources for $B$. bituminosa genetic analysis and breeding.

\section{Results}

\section{Sampling the $B$. bituminosa leaf transcriptome by Roche 454 sequencing}

Sequencing of the B. bituminosa leaf-derived cDNA library on the GS-FLX System resulted in 432,306 sequence reads with an average length of 345 bp (149.1 $\mathrm{Mbp}$ ). These sequence reads were deposited at the Sequence Read Archive (SRA) database at NCBI [GenBank:SRA037309]. GS De Novo Assembler software assembled 266,461 (61.6\%) of the reads into 4,542 contigs that were $\geq 100 \mathrm{bp}$ in length. It then grouped contigs into 2,929 "isogroups" (analogous to genes) and 3,798 "isotigs" (analogous to transcripts) with an average isotig length of 707 base pairs. 3657 isotigs and 
remaining singleton contig sequences that were $\geq 200$ bp in length were deposited at NCBI Transcriptome Shotgun Assembly (TSA) database [GenBank:JL856153JL859809].

\section{Functional characterisation of expressed gene sequences}

3,838 isotigs and remaining singleton contig sequences $\geq$ $100 \mathrm{bp}$ were subjected to in silico functional characterisation using Blast2GO software. Gene ontology (GO) terms were identified for 3,419 sequences (89.1\%; Additional file 1 ). Figure 1 provides a summary of the main GO terms defined according to the cellular component, biological process and molecular function associated with these 3,419 sequences. The species that received the greatest proportion of BLAST hits was Glycine max (3,013 hits, or $88.1 \%)$, and G. $\max$ was also the species that was most frequently the top hit (for 1,454 sequences; $42.5 \%$ ). The other main model legume species only rarely provided the most significant matches: Medicago truncatula was the top hit for 185 sequences (5.4\%) and Lotus japonicus was the top hit for 14 sequences $(0.4 \%)$. Together, these results indicate that G. $\max$ will serve as the most representative reference genome for $B$. bituminosa.

\section{SSR primer design and testing}

With the aid of QDD software, primer pairs for 186 'perfect' SSRs and 54 'compound' SSRs were designed (Additional file 2). Of these, 49 primer pairs for perfect SSRs and 38 primers pairs for compound SSRs were selected in descending order of SSR motif length along with a further 7 primer pairs were designed manually with the aid of Geneious software using contigs containing SSR motifs (Additional file 3). In total, 94 primer pairs were synthesised and screened for amplification efficiency using genomic DNA from $B$. bituminosa plant A13.1 as template. Without any specific optimisation, 82 out of 94 primer pairs (87.2\%) amplified one or more bands (visualised on low-resolution 1\% agarose gels), with one band being more common (78 primer pairs, $83.0 \%$ ) than $\geq 2$ bands ( 4 primer pairs, $4.3 \%$ ) (Additional file 3).

The 82 primer pairs that gave amplification products were screened for gross-level polymorphism in eight diverse $B$. bituminosa accessions (one plant per accession) by electrophoresis using $2 \%$ agarose gels. Of the 82 primer pairs tested in this way, 21 showed clear band size polymorphism and were selected for synthesis of fluorescently labelled forward primers (Table 1). These 21 fluorescently labelled primer pairs were used to genotype 79 B. bituminosa accessions (one plant per accession). These accessions were classed as 'original populations' (collected from wild or from traditional pasture lands; $\mathrm{n}=27$ ) or 'breeding lines' (having
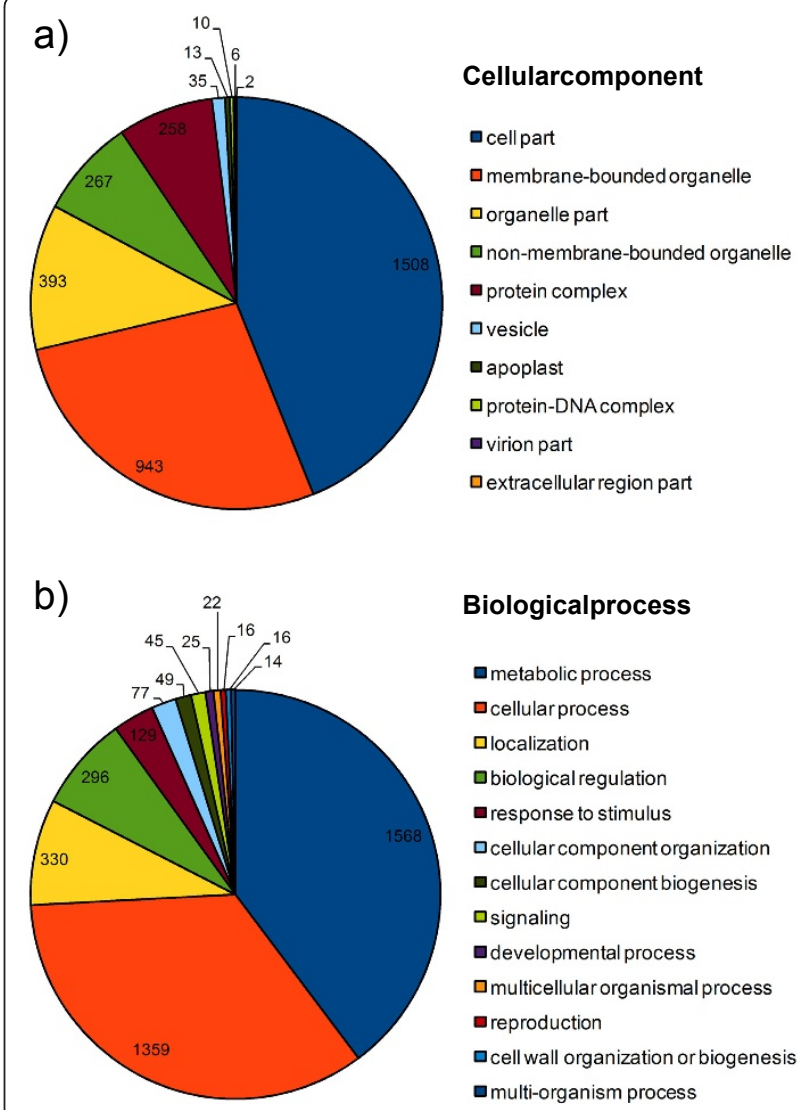

Biologicalprocess

- metabolic process

- cellularprocess

口localization

$\square$ biological regulation

- response to stimulus

$\square$ cellular component organization

- cellular component biogenesis

口signaling

-developmental process

口multicellular organismal process

- reproduction

- cell wall organization or biogenesis

-multi-organism process

c)

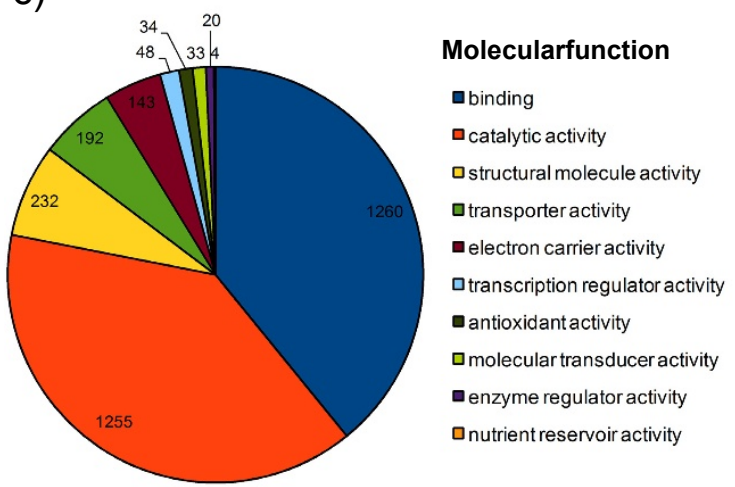

Figure 1 Gene ontology (GO) terms for 3,419 expressed gene sequences obtained by Roche 454 sequencing of leaf mRNA of Bituminaria bituminosa plant 13.1. GO descriptors are categorised according to: a) cellular components (level 3 terms); b) biological process (level 2 terms with > 10 sequences); and c) molecular function (level 2 terms)

undergone selection and possible uncontrolled crosspollination within the Spanish breeding programme; $\mathrm{n}=$ 52) (Table 2).

Fragment analysis using GeneMarker software revealed that 20 primer pairs gave clear peaks; the remaining primer pair gave variable amplification 
Table 1 Bituminaria bituminosa simple sequence repeat (Bbit-SSR) primer pairs selected for characterisation of $B$. bituminosa germplasm

\begin{tabular}{|c|c|c|}
\hline Marker name & Forward primer sequence & Reverse primer sequence \\
\hline Bbit-SSR004 & ACCACCCGCAGTTACTTTCACCT & CCTTGTGCTGGTTTCACGCAACG \\
\hline Bbit-SSR005 & ACCAAGTCAGGCTGGAACCCCA & GTCCTGGCCCACTGAACGCC \\
\hline Bbit-SSR008 & CATTGACATCCCTAAGCATAATGT & TCGTTAATAGCGGTCTTGGG \\
\hline Bbit-SSR010 & GCAGGCTTTCCTGAACTGAC & GTCTCCACCAGCAATACCGT \\
\hline Bbit-SSR012 & TCATCCСTTCTCTTCCTACTCG & CGGTTTCTTCGAATACACAGTA \\
\hline Bbit-SSR013 & GAAGGCAAGTGAAAAGCCAG & TCAGACACCAGTGGCTCAAC \\
\hline Bbit-SSR015 & GACTGCACGGTCTTCTCGAC & ATGTGCAGAGGCATTTGTTG \\
\hline Bbit-SSR034 & CAATCCCATTTTCCGCTTTA & TGCCCTCTTCCTTCATAGGTT \\
\hline Bbit-SSR035 & ATATCCACCACCTTCCGTGA & GTAGGATAGGGTCCGGTGGT \\
\hline Bbit-SSR040 & TAACCACTTGGAACTGGGGT & AATTGCAACAGCAGCAACAG \\
\hline Bbit-SSR055 & AGCATCACTACGACCATCCC & GGTGACAACAGAGTGGTCTGA \\
\hline Bbit-SSR056 & TCATCCCTTCTCTTCCTACTCG & CGGTTTCTTCGAATACACAGTA \\
\hline Bbit-SSR059 & CATTGACATCCCTAAGCATAATGT & TCGTTAATAGCGGTCTTGGG \\
\hline Bbit-SSR064 & TTGCTTCTGCGTAACTGTGG & AAAAGTCCACGTCAGCATCC \\
\hline Bbit-SSR066 & GGTCGTCCCATTTATCGAAG & GGAAGAACGGTCAATGGAGA \\
\hline Bbit-SSR067 & TCACCTTCCTCACAAACTACCA & TGAAATGCCTCAATGAGCTAAA \\
\hline Bbit-SSR070 & TGTCGAACTGTTGGATTGTGA & AATTGCAACAGCAGCAACAG \\
\hline Bbit-SSR073 & TTGCTTGTGTCCTGTCCAA & ССTTCCCTTACCCACCAAGT \\
\hline Bbit-SSR076 & AGAAGGCAAGTGAAAAGCCA & TCAGACACCAGTGGCTCAAC \\
\hline Bbit-SSR079 & GAGCTTCGGAGGGAGTTCTT & CCAAAATCCATCACCTTCCA \\
\hline Bbit-SSR090 & CCCTAACATTGGTAACAGCCA & GAGGCTGGCATCAAGTCAAC \\
\hline
\end{tabular}

strength and was consequently omitted from subsequent analysis. Of the 20 high-quality SSR primer pairs, 19 appeared to detect single loci ( 1 to 2 alleles per primer pair) while one primer pair appeared to detect two loci ( 2 to 4 alleles for primer pair 'Bbit-SSR079'). In total, 130 alleles were detected at 21 high-quality marker loci, an average of 6.19 alleles per locus indicating that these markers were generally highly polymorphic. The 19 single locus SSR markers detected between 3 to 11 alleles per marker, with polymorphic index content (PIC) values ranging from 0.13 to 0.76 (average $=0.407$ ) (Additional file 4).

Validating SSR markers by genetic diversity analysis in 27 original populations

To determine if the SSR markers were suitable for inferring genetic relationships among breeding lines, a pairwise Euclidean distance matrix for 27 accessions collected from the wild or from traditional pasturelands (termed 'original populations'; Table 2) was analysed by hierarchical clustering and MDS analysis (Figure 2). In both analyses, accessions grouped together according to botanical variety indicating that allelic variation at these markers reflected well-established botanical varieties. Interestingly, var. bituminosa accessions were sub-divided into two clear groups according to geographical origin (Canary Islands and Mediterranean region). Cluster analysis revealed that some accessions were genetically so similar that they could not be distinguished using allele information at 21 SSR loci (Figure 2a). Heterozygosity of the original populations ranged from $0-62 \%($ mean $=16.4 \%$; Table 2$)$. An analysis of molecular variance (AMOVA) confirmed that there was significant differentiation between populations accounting for $35 \%$ of the total allelic variance observed (Table 3).

\section{Using markers to characterise $B$. bituminosa breeding lines}

Having established that the usefulness of the new SSR markers, genotype information from an additional $52 \mathrm{~B}$. bituminosa breeding lines was subjected to pairwise distance analysis, along with plants from the 27 original populations (Additional file 5). This distance matrix was analysed by MDS and hierarchical clustering techniques (Figure 3 and Additional file 6). Adding these lines to the analyses resulted in an increased complexity of inter-relationships as evidenced by an increase in the two-dimensional MDS stress from 0.16 (27 original populations) to 0.23 (all 79 accessions). However, a similar pattern of groupings among the original populations was observed (compare Figure 2b and Figure 3). 
Table 2 Single Bituminaria bituminosa plants sampled from 79 accessions from original populations $(n=27)$ or breeding lines $(n=52)$

\begin{tabular}{|c|c|c|c|c|}
\hline Accession code $^{1}$ & Botanical variety & Geographical origin & Population type $^{2}$ & Heterozygosity $^{3}$ \\
\hline $\mathrm{A} 1.1$ & albomarginata & $\begin{array}{l}\text { Canary Islands } \\
\text { (Famara, Lanzarote) }\end{array}$ & Breeding line & $24 \%$ \\
\hline $\bar{A} 2.1$ & albomarginata & Canary Islands & Breeding line & $24 \%$ \\
\hline A3.2 & albomarginata & Canary Islands & Breeding line & $25 \%$ \\
\hline$\overline{A 4.2}$ & albomarginata & Canary Islands & Breeding line & $5 \%$ \\
\hline$\overline{A 5.1}$ & albomarginata & $\begin{array}{l}\text { Canary Islands } \\
\text { (Famara, Lanzarote) }\end{array}$ & Breeding line & $10 \%$ \\
\hline A6.2 & bituminosa & $\begin{array}{l}\text { Canary Islands } \\
\text { (Teno, Tenerife) }\end{array}$ & Breeding line & $33 \%$ \\
\hline A7.2 & bituminosa & $\begin{array}{l}\text { Canary Islands } \\
\text { (Teno, Tenerife) }\end{array}$ & Breeding line & $24 \%$ \\
\hline A8.1 & albomarginata & Canary Islands & Breeding line & $15 \%$ \\
\hline$\overline{A 9.1}$ & albomarginata & $\begin{array}{l}\text { Canary Islands } \\
\text { (Famara, Lanzarote) }\end{array}$ & Breeding line & $5 \%$ \\
\hline A10.2 & albomarginata & Canary Islands & Breeding line & $0 \%$ \\
\hline $\bar{A} 11.17$ & albomarginata & $\begin{array}{l}\text { Canary Islands } \\
\text { (Famara, Lanzarote) }\end{array}$ & Breeding line & $14 \%$ \\
\hline$\overline{A 12.3}$ & bituminosa & $\begin{array}{l}\text { Canary Islands } \\
\text { (Teno, Tenerife) }\end{array}$ & Original population & $0 \%$ \\
\hline A13.1 & bituminosa & $\begin{array}{l}\text { Canary Islands } \\
\text { (Teno, Tenerife) }\end{array}$ & Breeding line & $29 \%$ \\
\hline$\overline{\mathrm{A} 14.1}$ & albomarginata & $\begin{array}{l}\text { Canary Islands } \\
\text { (Malpaso, Lanzarote) }\end{array}$ & Original population & $19 \%$ \\
\hline$\overline{\mathrm{A} 15.4}$ & albomarginata & $\begin{array}{l}\text { Canary Islands } \\
\text { (Famara, Lanzarote) }\end{array}$ & Original population & $10 \%$ \\
\hline$\overline{A 16.2}$ & crassiuscula & $\begin{array}{l}\text { Canary Islands } \\
\text { (Vilaflor, Tenerife) }\end{array}$ & Breeding line & $10 \%$ \\
\hline $\bar{A} 17.2$ & crassiuscula & Canary Islands (Cañadas del Teide, Tenerife) & Breeding line & $12 \%$ \\
\hline$\overline{A 18.8}$ & albomarginata & $\begin{array}{l}\text { Canary Islands } \\
\text { (Vinamar, Fuerteventura) }\end{array}$ & Original population & $0 \%$ \\
\hline $\bar{A} 19.3$ & crassiuscula & $\begin{array}{l}\text { Canary Islands } \\
\text { (Tenerife) }\end{array}$ & Breeding line & $24 \%$ \\
\hline $\bar{A} 20.1$ & albomarginata & $\begin{array}{l}\text { Canary Islands } \\
\text { (Famara, Lanzarote) }\end{array}$ & Breeding line & $19 \%$ \\
\hline $\mathrm{A} 21.1$ & albomarginata & $\begin{array}{l}\text { Canary Islands } \\
\text { (Famara, Lanzarote) }\end{array}$ & Breeding line & $38 \%$ \\
\hline $\bar{A} 22.2$ & albomarginata & $\begin{array}{l}\text { Canary Islands } \\
\text { (Famara, Lanzarote) }\end{array}$ & Original population & $0 \%$ \\
\hline A23.2 & bituminosa & $\begin{array}{l}\text { Canary Islands } \\
\text { (Teno, Tenerife) }\end{array}$ & Original population & $28 \%$ \\
\hline A24.3 & bituminosa & $\begin{array}{l}\text { Canary Islands } \\
\text { (Teno, Tenerife) }\end{array}$ & Original population & $0 \%$ \\
\hline $\bar{A} 26.1$ & bituminosa & $\begin{array}{l}\text { Canary Islands } \\
\text { (Tenerife) }\end{array}$ & Original population & $25 \%$ \\
\hline A27.2 & crassiuscula & $\begin{array}{l}\text { Canary Islands } \\
\text { (Teide, Tenerife) }\end{array}$ & Original population & $33 \%$ \\
\hline $\bar{A} 29.2$ & albomarginata & $\begin{array}{l}\text { Canary Islands } \\
\text { (Famara, Lanzarote) }\end{array}$ & Original population & $6 \%$ \\
\hline$\overline{\mathrm{A} 36.1}$ & albomarginata & $\begin{array}{l}\text { Canary Islands } \\
\text { (Famara, Lanzarote) }\end{array}$ & Original population & $27 \%$ \\
\hline A37.2 & albomarginata & $\begin{array}{l}\text { Canary Islands } \\
\text { (Malpaso, Lanzarote) }\end{array}$ & Original population & $27 \%$ \\
\hline
\end{tabular}


Table 2 Single Bituminaria bituminosa plants sampled from 79 accessions from original populations $(n=27)$ or breeding lines $(\mathbf{n}=\mathbf{5 2})$ (Continued)

\begin{tabular}{|c|c|c|c|c|}
\hline A38.3 & bituminosa & $\begin{array}{l}\text { Canary Islands } \\
\text { (Tefia, Fuerteventura) }\end{array}$ & Original population & $14 \%$ \\
\hline A39.2 & albomarginata & $\begin{array}{l}\text { Canary Islands } \\
\text { (Vinamar, Fuerteventura) }\end{array}$ & Original population & $0 \%$ \\
\hline A40.1 & bituminosa & $\begin{array}{l}\text { Canary Islands } \\
\text { (Bentacuria, Fuerteventura) }\end{array}$ & Original population & $21 \%$ \\
\hline A41.2 & bituminosa & $\begin{array}{l}\text { Canary Islands } \\
\text { (Güimar, Tenerife) }\end{array}$ & Original population & $5 \%$ \\
\hline A43.2 & bituminosa & $\begin{array}{l}\text { Canary Islands } \\
\text { (Tenerife) }\end{array}$ & Breeding line & $38 \%$ \\
\hline A44.1 & albomarginata & Canary Islands & Breeding line & $10 \%$ \\
\hline A46.1 & albomarginata & Canary Islands & Breeding line & $10 \%$ \\
\hline $\bar{A} 48.2$ & albomarginata & $\begin{array}{l}\text { Canary Islands } \\
\text { (Famara, Lanzarote) }\end{array}$ & Breeding line & $52 \%$ \\
\hline A49.3 & crassiuscula & Canary Islands & Breeding line & $52 \%$ \\
\hline$\overline{\mathrm{A} 50.1}$ & albomarginata & $\begin{array}{l}\text { Canary Islands } \\
\text { (Famara, Lanzarote) }\end{array}$ & Breeding line & $15 \%$ \\
\hline A51.2 & crassiuscula & Canary Islands & Breeding line & $19 \%$ \\
\hline A52.1 & albomarginata & Canary Islands & Breeding line & $20 \%$ \\
\hline A53.2 & albomarginata & Canary Islands & Breeding line & $38 \%$ \\
\hline A54.1 & albomarginata & Canary Islands & Breeding line & $29 \%$ \\
\hline A55.2 & albomarginata & Canary Islands & Breeding line & $35 \%$ \\
\hline A56.2 & bituminosa & $\begin{array}{l}\text { Canary Islands } \\
\text { (Teno, Tenerife) }\end{array}$ & Breeding line & $37 \%$ \\
\hline$\overline{A 58.2}$ & albomarginata & $\begin{array}{l}\text { Canary Islands } \\
\text { (Famara, Lanzarote) }\end{array}$ & Breeding line & $52 \%$ \\
\hline A62.1 & albomarginata & Canary Islands & Breeding line & $43 \%$ \\
\hline$\overline{A 63.1}$ & crassiuscula & $\begin{array}{l}\text { Canary Islands } \\
\text { (Vilaflor, Tenerife) }\end{array}$ & Breeding line & $10 \%$ \\
\hline A64.2 & bituminosa & Canary Islands & Breeding line & $43 \%$ \\
\hline A65.1 & bituminosa & $\begin{array}{l}\text { Mediterranean region } \\
\text { (Calnegre, Murcia, Spain) }\end{array}$ & Breeding line & $0 \%$ \\
\hline $\mathrm{s} 1 \mathrm{~b}$ & bituminosa & Mediterranean region (Llano del Beal, Murcia, Spain) & Breeding line & $95 \%$ \\
\hline $\mathrm{S3C}$ & bituminosa & $\begin{array}{l}\text { Canary Islands } \\
\text { (Tahonilla, Tenerife) }\end{array}$ & Original population & $29 \%$ \\
\hline S4a & bituminosa & $\begin{array}{l}\text { Canary Islands } \\
\text { (Tahonilla, Tenerife) }\end{array}$ & Original population & $42 \%$ \\
\hline S6c & bituminosa & $\begin{array}{l}\text { Mediterranean region } \\
\text { (La Perdiz, Murcia, Spain) }\end{array}$ & Breeding line & $48 \%$ \\
\hline S8a & albomarginata & $\begin{array}{l}\text { Canary Islands } \\
\text { (Famara, Lanzarote) }\end{array}$ & Original population & $62 \%$ \\
\hline s9a & bituminosa & $\begin{array}{l}\text { Mediterranean region } \\
\text { (Calnegre, Murcia, Spain) }\end{array}$ & Breeding line & $68 \%$ \\
\hline $\mathrm{s} 10 \mathrm{~b}$ & bituminosa & $\begin{array}{l}\text { Mediterranean region } \\
\text { (Sardinia, Italy) }\end{array}$ & Original population & $24 \%$ \\
\hline $\mathrm{s} 11 \mathrm{~b}$ & bituminosa & $\begin{array}{l}\text { Mediterranean region } \\
\text { (La Unión, Murcia, Spain) }\end{array}$ & Breeding line & $62 \%$ \\
\hline$S 13 c$ & bituminosa & $\begin{array}{l}\text { Mediterranean region } \\
\text { (Calnegre, Murcia, Spain) }\end{array}$ & Breeding line & $30 \%$ \\
\hline $\mathrm{s} 14 \mathrm{~b}$ & bituminosa & $\begin{array}{l}\text { Mediterranean region } \\
\text { (Israel) }\end{array}$ & Original population & $33 \%$ \\
\hline $\mathrm{S} 17 \mathrm{~b}$ & bituminosa & $\begin{array}{l}\text { Mediterranean region } \\
\text { (La Unión, Murcia, Spain) }\end{array}$ & Original population & $0 \%$ \\
\hline
\end{tabular}


Table 2 Single Bituminaria bituminosa plants sampled from 79 accessions from original populations $(\mathrm{n}=27)$ or breeding lines $(\mathbf{n}=\mathbf{5 2})$ (Continued)

\begin{tabular}{|c|c|c|c|c|}
\hline $\mathrm{S} 18 \mathrm{~b}$ & bituminosa & $\begin{array}{l}\text { Mediterranean region } \\
\text { (La Unión, Murcia, Spain) }\end{array}$ & Original population & $5 \%$ \\
\hline S19b & bituminosa & $\begin{array}{l}\text { Mediterranean region } \\
\text { (La Unión, Murcia, Spain) }\end{array}$ & Original population & $5 \%$ \\
\hline $\mathrm{S} 20 \mathrm{~b}$ & bituminosa & $\begin{array}{l}\text { Mediterranean region } \\
\text { (La Perdiz, Murcia, Spain) }\end{array}$ & Breeding line & $48 \%$ \\
\hline $\mathrm{S} 21 \mathrm{C}$ & albomarginata & $\begin{array}{l}\text { Canary Islands } \\
\text { (Famara, Lanzarote) }\end{array}$ & Breeding line & $67 \%$ \\
\hline $\mathrm{S} 23 \mathrm{~b}$ & bituminosa & $\begin{array}{l}\text { Mediterranean region } \\
\text { (La Perdiz, Murcia, Spain) }\end{array}$ & Breeding line & $95 \%$ \\
\hline $\mathrm{S} 29 \mathrm{~b}$ & bituminosa & $\begin{array}{l}\text { Canary Islands } \\
\text { (Tenerife) }\end{array}$ & Breeding line & $15 \%$ \\
\hline S30b & bituminosa & $\begin{array}{l}\text { Canary Islands } \\
\text { (Tenerife) }\end{array}$ & Breeding line & $0 \%$ \\
\hline S31b & bituminosa & $\begin{array}{l}\text { Mediterranean region } \\
\text { (Spain) }\end{array}$ & Breeding line & $50 \%$ \\
\hline S32C & bituminosa & $\begin{array}{l}\text { Canary Islands } \\
\text { (Tenerife) }\end{array}$ & Breeding line & $29 \%$ \\
\hline S33b & albomarginata & Canary Islands & Breeding line & $24 \%$ \\
\hline S34C & bituminosa & $\begin{array}{l}\text { Mediterranean region } \\
\text { (La Perdiz, Murcia, Spain) }\end{array}$ & Breeding line & $38 \%$ \\
\hline S35c & bituminosa & $\begin{array}{l}\text { Mediterranean region } \\
\text { (La Perdiz, Murcia, Spain) }\end{array}$ & Breeding line & $63 \%$ \\
\hline S36a & bituminosa & $\begin{array}{l}\text { Mediterranean region } \\
\text { (La Perdiz, Murcia, Spain) }\end{array}$ & Breeding line & $35 \%$ \\
\hline S37c & bituminosa & $\begin{array}{l}\text { Mediterranean region } \\
\text { (La Perdiz, Murcia, Spain) }\end{array}$ & Breeding line & $55 \%$ \\
\hline S38d & bituminosa & $\begin{array}{l}\text { Mediterranean region } \\
\text { (Greece) }\end{array}$ & Original population & $11 \%$ \\
\hline S39c & bituminosa & $\begin{array}{l}\text { Mediterranean region } \\
\text { (Greece) }\end{array}$ & Original population & $5 \%$ \\
\hline s40d & bituminosa & $\begin{array}{l}\text { Mediterranean region } \\
\text { (Greece) }\end{array}$ & Original population & $11 \%$ \\
\hline S41C & bituminosa & $\begin{array}{l}\text { Canary Islands } \\
\text { (La Palma) }\end{array}$ & Breeding line & $28 \%$ \\
\hline
\end{tabular}

${ }^{1}$ Accession names prefixed by ' $\mathrm{A}$ ' were provided by the Australian breeding programme, while accession names prefixed by ' $\mathrm{S}$ ' were provided by the Spanish breeding programme.

${ }^{2}$ The term 'breeding line' indicates that the accession has been grown for one or more generation in breeding nurseries with possible cross-pollination among accessions. The term 'original population' indicates that seed was collected directly from geographical location indicated with no opportunity for cross-pollination among these accessions.

${ }^{3}$ Heterozygosity was assessed at 21 simple sequence repeat loci.

Figure 3a-3d shows the placement of breeding lines of three botanical varieties from the Canary Islands and Mediterranean region relative to the original populations. In general, breeding lines described as var. albomarginata and var. bituminosa (from the Canary Islands) were placed within or near their respective clusters of original populations with one notable exception: breeding line S41c (Figure 3b) was described as Canary Island bituminosa but had much greater affinity to Mediterranean bituminosa according to SSR marker genotyping. Breeding lines described as Mediterranean bituminosa and var. crassiuscula had very wide distribution in the MDS plots (Figure 3c and 3d). The average heterozygosity of breeding lines was substantially higher (32.0\%) than for original populations (16.4\%), and had a greater range of values (0-95\%; Table 2$)$.

\section{Discussion}

New high-throughput DNA sequencing technologies offer many research opportunities for both model and crop species alike [15]. Arguably, minor grain and pasture crop species (also known as "orphan" crop species [19]) stand to gain the most since they are starting from a very low baseline of genomic resources. This paper describes a method for developing codominant and polymorphic genic SSR markers from a modest 

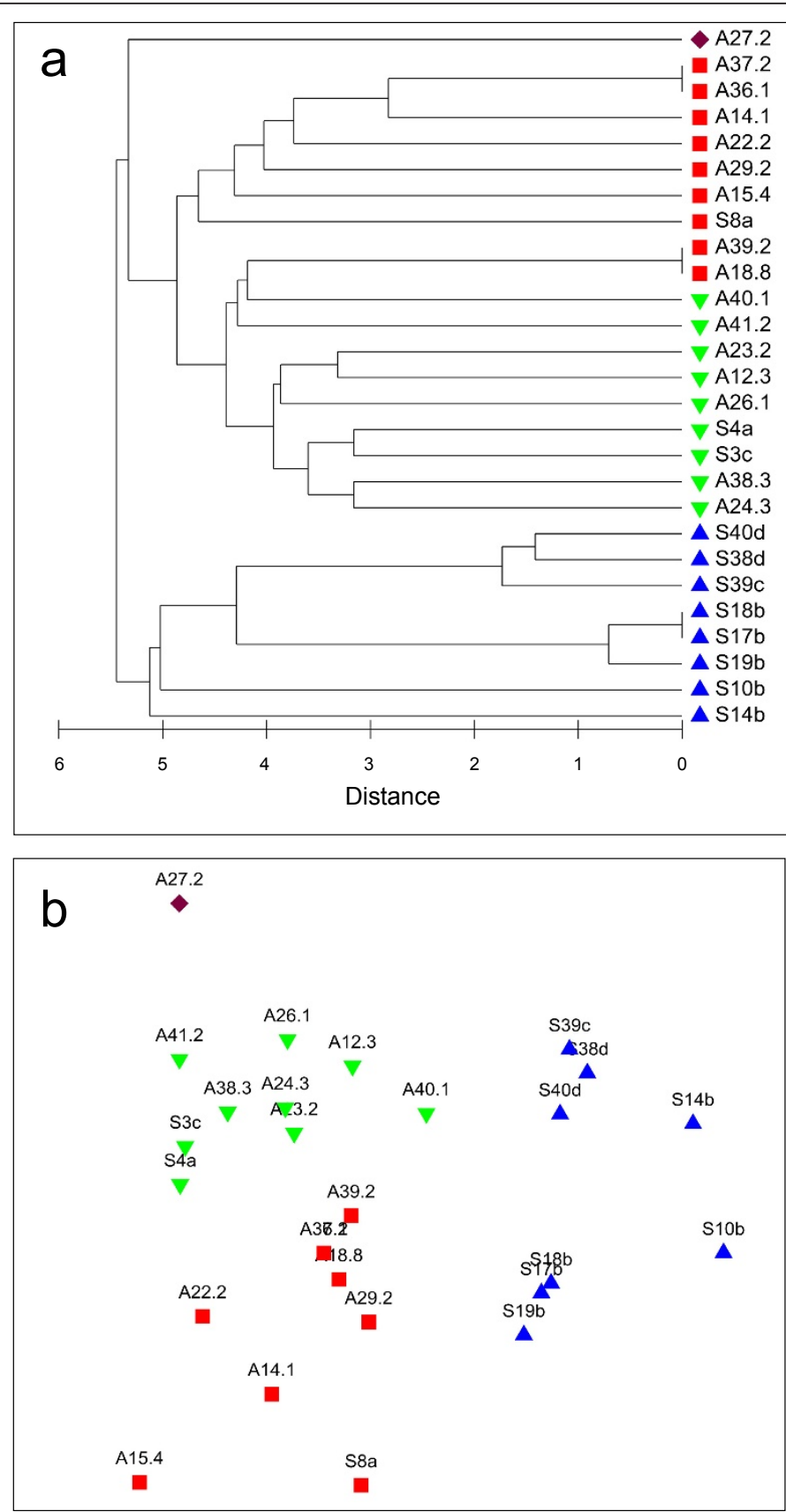

albomarginata

$\checkmark$ Canary bituminosa

- Mediterranean bituminosa

crassiuscula

Figure 2 Genetic relationships among 27 Bituminaria bituminosa accessions from three botanical varieties collected from the Canary Islands and the Mediterranean region. One plant from each accession was assessed using 21 simple sequence repeat loci with the resulting 130 alleles used to calculate Euclidean pairwise distances. These distances are presented in: a) Dendrogram produced by hierarchical clustering analysis; b) Multi-dimensional scaling (MDS) plot (2D stress $=0.16)$. 
Table 3 Analysis of molecular variance (AMOVA) in single plants sampled from 26 original populations of Bituminaria bituminosa

\begin{tabular}{cccccc}
\hline Source & df & SS & MS & Est. Var. & $\%$ \\
\hline Between Pops & 2 & 180.425 & 90.213 & $8.549^{*}$ & $35 \%$ \\
\hline Within Pops & 23 & 373.306 & 16.231 & $16.231^{*}$ & $65 \%$ \\
\hline Total & 25 & 553.731 & & 24.780 & $100 \%$
\end{tabular}

*Significant at $P<0.001$

Three populations were defined as var. albomarginata $(n=9)$, var. bituminosa from the Canary Islands $(n=9)$ and var. bituminosa from the Mediterranean region $(n=8)$. Botanical variety crassiuscula was not included because there was only one accession used in this study.

sampling of the leaf transcriptome derived from a single plant of B. bituminosa by Roche 454 pyrosequencing. This method was highly effective with 75 out of 87 (86.2\%) automatically designed primer pairs successfully amplifying PCR products (Additional file 3). Of the 21 markers selected for high- resolution fragment analysis in plants from 79 B. bituminosa accessions, 20 gave consistently strong amplification products and were highly polymorphic (6.19 alleles per marker locus). The methods presented in this study could be used by researchers for other genomic orphan species for rapid development of high-quality codominant markers, although the extent of marker polymorphism will vary between species and between populations within species.

The usefulness of the SSR markers was demonstrated by a clear delineation of groups according to botanical variety and geographical location in plants sampled from 27 original populations. These markers advanced our understanding of genetic diversity in B. bituminosa in that we could clearly distinguish var. bituminosa types according to their geographical origin (Canary Islands and the Mediterranean region; Figure 2). This differentiation was of similar magnitude to that which distinguished botanical varieties and extends the observation by Juan et al. [4] who found that Mediterranean var. bituminosa formed its own grouping while all botanical varieties from the Canary Islands formed another group. Therefore, there is a prima facie case for dividing var. bituminosa into two botanical varieties according to geographic origin (Canary Island or Mediterranean region), each distinct from var. albomarginata and var. crassiuscula. There are some differences in trait characteristics that support this division. For example, Mediterranean var. bituminosa are usually biennial whereas Canary Island var. bituminosa are perennial.

These SSR markers provided some useful insights into $B$. bituminosa breeding lines. Historically, the B. bituminosa breeding programme has practiced uncontrolled open pollination. While most pollination in B. bituminosa is considered to be by self-pollination, a certain proportion of out-crossing does occur [21] but until now there has been little direct evidence to support this assumption. In this study, two lines of evidence were found to support the occurrence of out-crossing in the B. bituminosa breeding programme. First, the average level of heterozygosity increased approximately two-fold in breeding lines $(32.0 \%)$ versus original populations (16.4\%) (Table 2). Second, cross-pollination between $B$. bituminosa plants from different botanical varieties and geographical locations is the most likely explanation of the wider distribution of breeding lines compared to original populations in MDS plots (Figure 3). Interestingly, this increased distribution was most pronounced in Mediterranean var. bituminosa. This may in part be explained by breeder observations in Murcia (Spain) that populations of var. bituminosa presented a higher percentage of fruit set compared to populations of var. albomarginata, which was taken to be indirect evidence of higher cross-pollination frequencies in var. bituminosa [22]. The same observation was made for var. crassiuscula, though analysis of additional accessions is necessary to confirm this preliminary conclusion.

An advantage of using the transcriptome sequencing approach compared to more conventional genomic SSR approaches is that markers should be more transferable across species since they are based on gene sequences that are relatively well conserved in evolution compared to non-genic regions [23]. For example, these genic markers may prove useful in other Psoraleeae species that remain genomic orphans, such as Cullen australasicum, an Australian native perennial legume species that shows promise as a drought-tolerant pasture species [24].

In addition to generating useful SSR markers, this study provides a repertoire of many thousands of expressed gene sequences for potential follow-up experiments. Gene ontology analysis using Blast2GO was highly effective in identifying putative cellular location, biological process and molecular function in 3,419 out of 3,838 (89.1\%) assembled mRNA sequences. Examples of follow-up experiments may include investigating genes associated with flowering time regulation (e.g. CONSTANS-like a [23]; isotig02726, Additional file 1) or furanocoumarin biosynthesis genes (e.g. cytochrome p450 monooxygenases [24,25]; e.g. isotig00288, Additional file 1). The species showing closest homology to B. bituminosa in 3,013 out of 3,419 (88.1\%) mRNA sequences with significant hits was G.max, indicating that G. $\max$ will serve as a useful reference genome for future gene discovery and characterisation in $B$. bituminosa.

\section{Conclusions}

This paper describes an efficient method for developing valuable SSR markers for B. bituminosa, a species that 

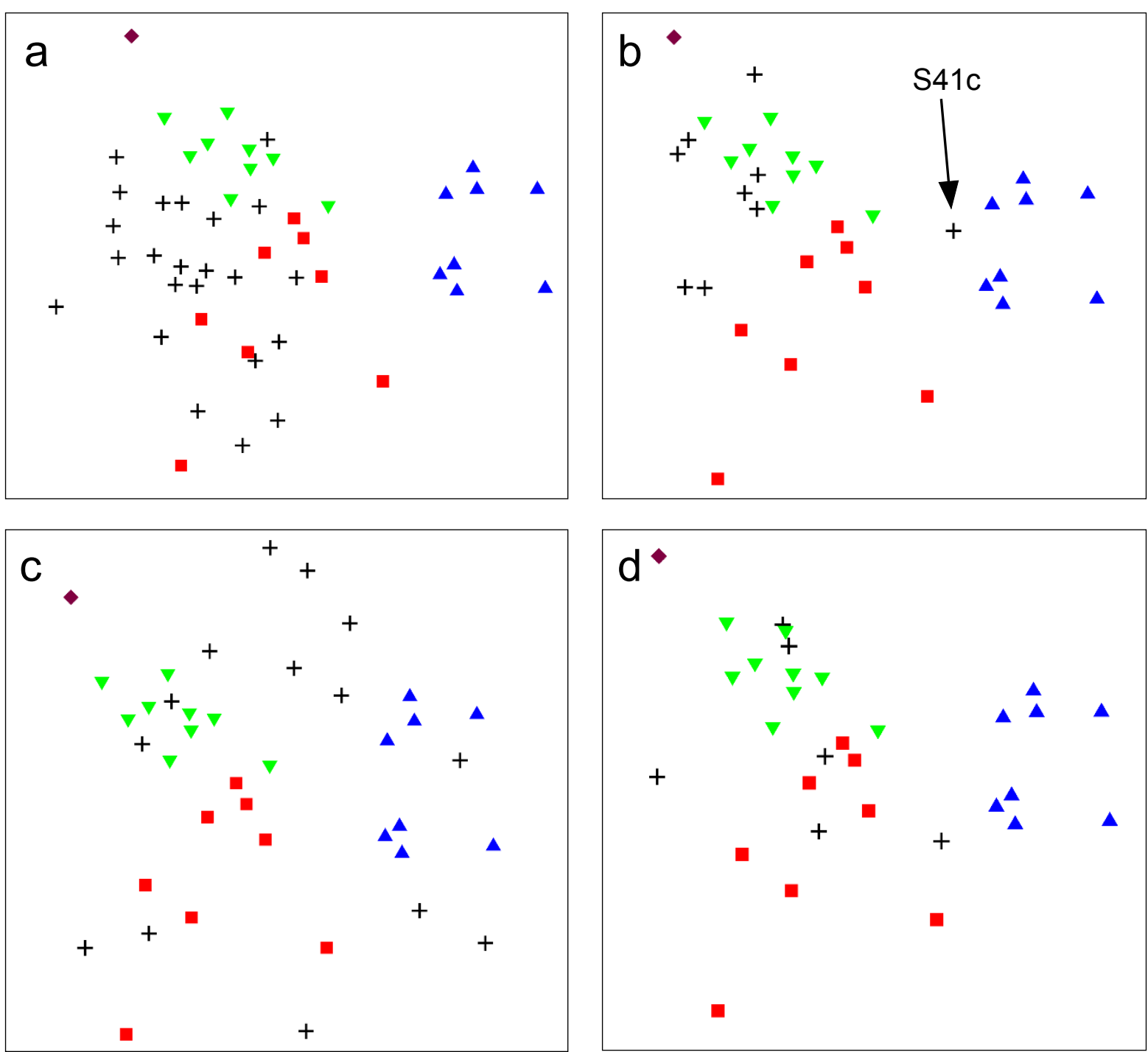

Botanical variety description of plants from original collections:

albomarginata

$\checkmark$ Canary bituminosa

- Mediterranean bituminosa

- crassiuscula

+ Breeding line

Figure 3 Multidimensional scaling (MDS) plots of 79 Bituminaria bituminosa accessions from three botanical varieties from the Canary Islands and the Mediterranean region. Each MDS plot was generated from pairwise Euclidean distances of all 79 accessions (2D stress = 0.23$)$ with each plot showing the position of additional breeding lines (denoted by " + " signs) from botanical varieties: a) var. albomarginata; b) var. bituminosa (Canary Islands); c) var. bituminosa (Mediterranean region); and d) var. crassiuscula.

could previously be described as a genomic orphan. These markers gave new insight into genetic variation in B. bituminosa, providing evidence that a division of the botanical variety bituminosa may be appropriate.
Evidence of cross pollination was found between botanical varieties in the $B$. bituminosa breeding programme. The expressed gene repertoire discovered in this experiment may be useful for follow-up experiments targeting 
biochemical pathways and/or important agronomic traits.

\section{Methods \\ Selection of a $B$. bituminosa accession for transcriptome sequencing \\ In June 2008, one plant from each of 22 accessions was analyzed for psoralen and angelicin content (Ewald Sweeny, Chem Centre, Western Australia; unpublished data). The plant A13.1 (from accession A13) had the highest total content of furanocoumarins (highest in angelicin and third highest in psoralen) out of the 22 plants evaluated. This accession is from an original population collected from the Teno region of the Can- ary Islands. Plant A13.1 was cloned by propagating cut- tings which were then kept at the Department of Agriculture and Food Western Australia (DAFWA) in a naturally lit glasshouse at a constant temperature of $25^{\circ}$ C. One such clone was used for transcriptome sampling.}

\section{mRNA extraction and cDNA preparation}

Young leaves were harvested from A13.1 and immediately frozen in liquid nitrogen. Leaves were ground to a fine powder in liquid nitrogen using a mortar and pestle. Total RNA was extracted using RNeasy kit (Qiagen) and mRNA isolated using Oligotex mRNA kit (Qiagen). mRNA was purified using RNeasy Minelute kit (Qiagen) and quality checked at the Australian Genome Research Facility (AGRF; Brisbane, Australia) using an RNA6000 Pico chip (Agilent) run on a BioAnalyzer 2100 (Agilent). A cDNA library was constructed by AGRF following the standard Roche Diagnostics protocol ("cDNA Rapid Library Preparation Method Manual-GS FLX Titanium Series", October 2009 (Rev. Jan 2010)).

\section{Sampling the $B$. bituminosa transcriptome by Roche 454 sequencing}

Sequencing of the cDNA library was carried out at AGRF using the GS-FLX System (Roche Diagnostics) with Titanium sequencing chemistry on one half of a two-region gasket PicoTitre Plate; for full details, consult the Roche Diagnostics "Sequencing Method Manual-GS FLX Titanium Series”, October 2009 (Rev. Jan 2010). The GS De Novo Assembler software (Roche Diagnostics) was used to assemble the sequencing output into contigs, using default parameters.

\section{De novo assembly of $B$. bituminosa leaf transcriptome}

The GS De Novo Assembler (version 2.3, Roche Diagnostics) software programs "newAssembly" (with "cdna" parameter) and "runProject" were used to align and assemble the sequencing output from Standard Flowgram Format into contigs and isotigs, using default parameters as described by the manufacturer.

\section{Functional Analysis}

Gene ontology (GO) classification was conducted with the aid of Blast2GO software [26] using GenBank database version 173 . Isotig and remaining singleton contigs $>100$ bp were included in the analysis. Matches with significance values $<1$ e- 6 were allocated to three GO categories (Biological Process, Molecular Function, and Cellular Component) in 1 to 11 levels of hierarchical structure. For ease of visualisation, results are presented at levels 2 or 3 (Figure 1).

\section{SSR detection and primer design}

The raw GS-FLX sequencing output in FASTA format was submitted as input to the QDD program [27] for detection of SSR markers and primer design. The QDD program was run from the command line on a Linux system using the default parameters as described in the QDD user manual. For comparison, a manual search for repeat motifs among the isotig/contig sequences was performed and primers flanking a subset of repeats were designed with the aid of Geneious 5.3 (Biomatters Ltd).

\section{SSR marker amplification and fragment analysis}

Genomic DNA was extracted from 50 B. bituminosa accessions provided by the Future Farm Industries Cooperative Research Centre (FFI CRC) at the Department of Agriculture and Food Western Australia (South Perth, Australia) and 29 B. bituminosa accessions provided by the Spanish Breeding programme at Instituto Murciano de Investigación y Desarrollo Agrario y Alimentario (Murcia, Spain) (Table 2), using Illustra Nucleon Phytopure Genomic DNA Extraction Kits (GE Healthcare). PCR reactions were carried out in a MasterCycler programmable thermal cycler (Eppendorf) in $20 \mathrm{uL}$ volumes containing the following components: $2.5 \mathrm{ng} / \mathrm{uL}$ genomic DNA, 1x PCR buffer (comprising 50 $\mathrm{mM} \mathrm{KCl}, 10 \mathrm{mM}$ Tris $\mathrm{HCl}$ (pH 9.0) and $0.1 \%$ Triton$\mathrm{X}), 2 \mathrm{mM} \mathrm{MgCl} 2,200 \mu \mathrm{M}$ dNTPs, 0.04 U/uL Taq DNA polymerase and $0.2 \mu \mathrm{M}$ each of forward and reverse primers. Amplification conditions consisted of denaturation at $94^{\circ} \mathrm{C}$ for 5 min followed by 35 cycles of denaturation at $94^{\circ} \mathrm{C}$ for $45 \mathrm{sec}$, primer annealing at $55^{\circ} \mathrm{C}$ for $45 \mathrm{sec}$ and extension at $72^{\circ} \mathrm{C}$ for $90 \mathrm{sec}$, followed by a final extension step of $72^{\circ} \mathrm{C}$ for $7 \mathrm{~min}$.

Initial screening of 96 SSR primer pairs for amplification efficiency was carried out using genomic DNA from plant A13.1 that had been used for transcriptome sequencing. Primer pairs that successfully amplified fragments in A13.1 (assessed using conventional TBE agarose electrophoresis) were then used to screen eight diverse B. bituminosa accessions (individual plants: A13.1, A27.2, A37.2, A42.3, A43.2, A48.2, A51.2 and S2b) for polymorphism. For those markers identified as polymorphic on $2 \%$ TBE agarose gels, 
fluorescently-labelled forward primers were synthesised and used to amplify fluorescently labelled amplicons in 79 lines. Fragment size analysis was performed relative to a Genescan LIZ500 internal size standard (Applied Biosystems) using an AB3730xl capillary DNA sequencer (Applied Biosystems) with the resulting electropherograms analysed using GeneMarker software (SoftGenetics) as described in detail by Nelson et al. [28]. Each marker allele was recorded as estimated base pair length. For genetic distance estimates, alleles were scored as present (1), absent (0) or unknown (999).

\section{Genetic diversity analyses}

Pairwise Euclidean distances among 79 B. bituminosa accessions (Table 2) were calculated using NTSYSpc 2.21i (Applied Biostatistics Inc.). Pairwise distances were subjected to hierarchical cluster analysis using group averages and multidimensional scaling (MDS) using Kruskal fit scheme 1 with 100 restarts in Primer 6.1.6 software (Primer-E Ltd). Analysis of Molecular Variance (AMOVA) of variation within and among original populations ( $\mathrm{n}=26$, excluding var. crassiuscula as there was only one accession represented) and allele frequencies $(\mathrm{n}=27)$ were calculated using GenAlEx 6.4 [29]. Polymorphism information content (PIC) was calculated using the formula described by Pradhan et al. [30].

\section{Additional material}

Additional file 1: Gene ontology (GO) terms for 3,419 Bituminaria bituminosa mRNA sequences. $\mathrm{GO}$ terms with associated descriptions generated using Blast2GO software.

Additional file 2: 240 primer pairs targeting simple sequence repeat motifs. Primer pairs designed using Primer3 based on Roche 454 sequences identified as containing simple sequence repeats using QDD software.

Additional file 3: Primer pairs selected for testing. Details of 94 primer pairs targeting simple sequence repeat (SSR) motifs in transcribed genes of Bituminaria bituminosa leaves sampled by Roche 454 transcriptome sequencing.

Additional file 4: Allele frequencies in Bituminaria bituminosa plants. A total of 118 simple sequence repeat (SSR) marker alleles were detected using 19 single-locus markers. Marker Bbit-SSR079 detected two loci and so was not included here. Polymorphic information content (PIC) and allele frequencies were calculated based on the whole population of 79 B. bituminosa plants. Allele frequencies are also presented for four original populations of botanical varieties albomarginata, bituminosa (from the Canary Islands and Mediterranean region) and crassiuscula that together comprised 27 plants.

Additional file 5: Euclidean distance matrix. 79 Bituminaria bituminosa plants were genotyped at 21 simple sequence repeat loci. The resulting 130 alleles were used to calculate pairwise Euclidean distances.

Additional file 6: Cluster tree of 79 Bituminaria bituminosa plants. Based on Euclidean distances estimated using 130 simple sequence repeat marker alleles. Symbols indicate botanical variety and type of line (original collection or breeding line).

\section{Acknowledgements}

We thank Future Farm Industries CRC, ICIA and IMIDA for providing the germplasm of Tedera. We also thank the Instituto Nacional de Investigación y Tecnología Agraria y Alimetaria (Project RTA 2007-00046-00-00) for funding the Spanish breeding programme and The Ministrario de Ciencia e Innovación (Project BFU2010-19599) for providing support for publication costs. MPN thanks IMIDA and Caja Murcia for funding travel to UWA, UWA for hosting the visit and Dr Leonor Ruiz for advice on SSR analyses. MD thanks the European Social Fund. We thank Caroline Snowball for preparing fluorescent PCR samples for fragment analysis. MNN thanks James Hane for advice on genomic data analysis and sequence submission to NCBI.

\section{Author details}

'Departamento de Biotecnología y Protección de cultivos, Instituto Murciano de Investigación y Desarrollo Agrario y Alimentario (IMIDA), C/Mayor s/n, 30150-La Alberca, Murcia, Spain. ${ }^{2}$ Departamento de Recursos Naturales, IMIDA. C/Mayor s/n, 30150-La Alberca, Murcia, Spain. ${ }^{3}$ Australian Genome Research Facility, Level 5 Gehrmann Laboratories, Research Road, University of Queensland, St Lucia, QLD 4072, Australia. ${ }^{4}$ Centre for Ecohydrology, The University of Western Australia, 35 Stirling Highway, Crawley, WA 6009, Australia. ${ }^{5}$ School of Plant Biology, Faculty of Natural and Agricultural Sciences, The University of Western Australia, 35 Stirling Highway, Crawley, WA 6009, Australia. 'Department of Agriculture and Food, Western Australia, South Perth, WA 6151, Australia. ${ }^{7}$ Future Farm Industries Cooperative Research Centre, The University of Western Australia, 35 Stirling Highway, Crawley, WA 6009, Australia.

\section{Authors' contributions}

MPN carried out all laboratory work, except for RNA and mRNA extractions for the sequenced library, which were performed by NT, MD, EC and DR collected and characterised B. bituminosa germplasm and selected plants for DNA and RNA sampling. KH selected and implemented bioinformatic analyses. MNN planned and supervised the laboratory work, with additional supervision provided by NT. MPN and MNN conducted genetic diversity analyses. All authors contributed to the final version of the manuscript.

Received: 26 August 2011 Accepted: 15 December 2011 Published: 15 December 2011

\section{References}

1. Juan A, Moñino I, Correal E, Méndez P, Crespo MB: Comparación de las tasas de fructificación de Bituminaria bituminosa (Leguminosae) bajo condiciones de cultivo en Canarias y la Península lbérica. Pastos y Ganadería Extensiva Actas XLIV Reunion Cientifica de la SEEP: 2004; Salamanca, Spain 111-115, Sociedad Espanola para el Estudio de los Pastos.

2. Stirton $\mathrm{CH}$ : Tribe 11. Psoraleeae (Benth.) Rydb. (1919). Advances in legume systematics Part 1 Raven RMPPH; 1981, 337-343.

3. Walker D, Moñino I, Correal E: Genome size in Bituminaria bituminosa (L.) CH Stirton (Fabaceae) populations: separation of "true" differences from environmental effects on DNA determination. Environmental and Experimental Botany 2006, 55(3):258-265.

4. Méndez P, Fernandez M: Interés forrajero de las variedades de Bituminaria bituminosa (L.) Stirton ("tedera") de Canarias. XXX Reun cient Soc Esp para el estud de los pastos; Donostia-San Sebastian 1990, 264-272.

5. Méndez $P$, Fernández M, Santos A: Variedades de Bituminaria bituminosa (L.) Stirton (Leguminosae) en el archipiélago canario. Pastos 1990, 20:157-166.

6. Muñoz A, Correal E: Bituminaria bituminosa (L.) Stirton, leguminosa de interes forrajero en la Cuenca Mediterranea: I. Situacion taxonomica, autoecologia y distribucion. XXXVIII Reunion Cientifica de la SEEP: 1998; Soria 87-91.

7. Juan A, Coca B, Crespo MB, Rios S, Correal E: Datos preliminares sobre variabilidad molecular poblacional en el agregado de Bituminaria bituminosa (Leguminosae). Producciones agroganaderas: Gestión eficiente y conservación del medio natural 2005, II:971-977.

8. Muñoz A, Ortiz-Dorda J, Correal E: Morphological and molecular characterization of Bituminaria bituminosa accessions from South-East Spain and the Canary Islands. Cahiers Options Mediter 2000, 45:103-107.

9. Maurich T, lorio M, Chimenti D, Turchi G: Erybraedin C and bitucarpin A, two structurally related pterocarpans purified from Bituminaria 
bituminosa, induced apoptosis in human colon adenocarcinoma cell lines MMR- and p53-proficient and -deficient in a dose-, time-, and structure-dependent fashion. Chem Biol Interact 2006, 159:104-116.

10. Maurich T, Pistelli L, Turchi G: Anti-clastogenic activity of two structurally related pterocarpans purified from Bituminaria bituminosa in cultured human lymphocytes. Mutat Res 2004, 561:75-81.

11. Pistelli L, Noccioli C, Appendina G, Bianchi F, Sterner O, Ballero M: Pterocarpans from Bituminaria morisiana and Bituminaria bituminosa. Phytochem 2003, 64:595-598.

12. Rosa A, Deiana M, Corona G, Atzeri A, Incani A, Appendin G, Dessì MA: Antioxidant properties of extracts and compounds from Psoralea morisiana. Eur J Lipid Sci Technol 2005, 107:521-529.

13. Innocenti G, Cappelletti E, Caporale G: Furanocoumarin contents in the vegetative organs of cultivated Psoralea species. Int J Pharmacog 1991, 29:141-146.

14. Innocenti G, Piovan A, Filippini R, Caniato R, Cappelletti E: Quantitative recovery of furanocoumarins from Psoralea bituminosa. Phytochem Analysis 1997, 8:84-86.

15. Martinez S, Correal E, Real D, Ortuño A, Del Río J: Bituminaria bituminosa: A source of furanocoumarins of pharmaceutical interest. In Drug Plants I. Edited by: AS Awaad JGVS. Houston: Studium Press, LLC; 2010:307-322.

16. Real D, Li GD, Clark S, Albertsen TO, Hayes RC, Denton MD, D'Antuono MF, Dear BS: Evaluation of perennial forage legumes and herbs in six Mediterranean environments. Chilean Journal of Agricultural Research 2011.

17. Real D, Verbyla A: Maximizing genetic gains using a "plant" model in the Tedera (Bituminaria bituminosa var. albomarginata and var. crassiuscula) breeding program in Australia. Options Méditerranéennes, A 2010, 92:87-96.

18. Collard BCY, Mackill DJ: Marker-assisted selection: an approach for precision plant breeding in the twenty-first century. Philosophical Transactions of the Royal Society B: Biological Sciences 2008, 363(1491):557-572.

19. Varshney RK, Close TJ, Singh NK, Hoisington DA, Cook DR: Orphan legume crops enter the genomics era! Current opinion in plant biology 2009, 12(2):202-210.

20. Egan AN, Crandall KA: Incorporating gaps as phylogenetic characters across eight DNA regions: Ramifications for North American Psoraleeae (Leguminosae). Molecular Phylogenetics and Evolution 2008, 46(2):532-546.

21. Stefanović S, Pfeil BE, Palmer JD, Doyle JJ: Relationships among phaseoloid legumes based on sequences from eight chloroplast regions. Systematic Botany 2009, 34:115-128.

22. Schmutz J, Cannon SB, Schlueter J, Ma J, Mitros T, Nelson W, Hyten DL, Song Q, Thelen JJ, Cheng J, et al: Genome sequence of the palaeopolyploid soybean. Nature 2010, 463(7278):178-183.

23. Hecht V, Foucher F, Ferrandiz C, Macknight R, Navarro C, Morin J, Vardy ME, Ellis N, Beltran JP, Rameau C, et al: Conservation of Arabidopsis flowering genes in model legumes. Plant Physiology 2005, 137(4):1420-1434.

24. Larbat R, Kellner S, Specker S, Hehn A, Gontier E, Hans J, Bourgaud F, Matern $U$ : Molecular cloning and functional characterization of psoralen synthase, the first committed monooxygenase of furanocoumarin biosynthesis. Journal of Biological Chemistry 2007, 282(1):542-554.

25. Larbat R, Hehn A, Hans J, Schneider S, Jugdé H, Schneider B, Matern U, Bourgaud F: Isolation and functional characterization of CYP71AJ4 encoding for the first P450 monooxygenase of angular furanocoumarin biosynthesis. Journal of Biological Chemistry 2009, 284(8):4776-4785.

26. Götz S, García-Gómez JM, Terol J, Williams TD, Nagaraj SH, Nueda MJ, Robles M, Talón M, Dopazo J, Conesa A: High-throughput functional annotation and data mining with the Blast2GO suite. Nucleic Acids Research 2008, 36(10):3420-3435.

27. Meglécz E, Costedoat C, Dubut V, Gilles A, Malausa T, Pech N, Martin J: QDD: a user-friendly program to select microsatellite markers and design primers from large sequencing projects. Bioinformatics 2010, 26:403-404.

28. Nelson M, Mason A, Castello M-C, Thomson L, Yan G, Cowling W: Microspore culture preferentially selects unreduced (2n) gametes from an interspecific hybrid of Brassica napus L. $\times$ Brassica carinata Braun. TAG Theoretical and Applied Genetics 2009, 119(3):497-505.

29. Peakall R, Smouse P: genalex6: genetic analysis in Excel. Population genetic software for teaching and research. Molecular Ecology Notes 2006, 6(1):288-295

30. Pradhan A, Nelson MN, Plummer JA, Cowling WA, Yan G: Characterization of Brassica nigra collections using simple sequence repeat markers reveals distinct groups associated with geographical location, and frequent mislabelling of species identity. Genome 2011, 54:50-63.

doi:10.1186/1471-2156-12-104

Cite this article as: Pazos-Navarro et al:: Next generation DNA sequencing technology delivers valuable genetic markers for the genomic orphan legume species, Bituminaria bituminosa. BMC Genetics 2011 12:104.

\section{Submit your next manuscript to BioMed Central and take full advantage of:}

- Convenient online submission

- Thorough peer review

- No space constraints or color figure charges

- Immediate publication on acceptance

- Inclusion in PubMed, CAS, Scopus and Google Scholar

- Research which is freely available for redistribution

Submit your manuscript at www.biomedcentral.com/submit
Biomed Central 